\title{
Double Papilla Graft with Amnion Membrane for Root Coverage of Isolated Recession
}

\author{
${ }^{1}$ Ruchi Srivastava, ${ }^{2}$ Zeba R Siddiqui, ${ }^{3}$ Rajesh Jhingran, ${ }^{4}$ Vivek K Bains
}

\begin{abstract}
Gingival recession is one of the most common soft tissue problems, especially when it hinders the esthetics of a patient. Gingival recession can further lead to root caries, sensitivity, and difficulty in plaque control. Moreover, exposed roots are prone to abrasion and erosion. Periodontal plastic surgery is one of the emerging fields, which helps to meet such soft tissue problems. Currently, a variety of techniques are being used for root coverage, like pedicle grafts, coronally advanced flap, free mucosal graft, epithelial connective tissue graft, guided tissue regeneration, and acellular dermal matrix. This article highlights a case that was successfully managed by double papilla graft technique in combination with amnion membrane for root coverage of Miller class II recession defect in the mandibular canine region. In this technique, the soft tissues adjacent to the recession area were positioned over the recession defect. The main advantage of this technique is that the flap remains attached at its base so that it retains its own blood supply during transfer to a new position. It also preserves the interdental papilla, thus preserving the gingival height. As an alternative to the epithelial connective tissue graft, amnion membrane has the advantage of no invasive palatal wound, regenerative potential, and improved esthetic results. This procedure is highly sensitive and had predictable results, with a clinically significant amount of root coverage.
\end{abstract}

Keywords: Amnion membrane, Double papilla technique, Recession, Root coverage.

How to cite this article: Srivastava R, Siddiqui ZR, Jhingran R, Bains VK. Double Papilla Graft with Amnion Membrane for Root Coverage of Isolated Recession. World J Dent 2016;7(4):213-216.

Source of support: Nil

Conflicts of interest: None

\section{INTRODUCTION}

The basic intention of any root coverage procedure is to achieve complete restoration of all periodontal structures in the area of root recession. Gingival recession is defined as the exposure of root surfaces by displacement of the gingival margin apical to the cementoenamel

\footnotetext{
${ }^{1}$ Reader, ${ }^{2}$ Postgraduate Student, ${ }^{3,4}$ Professor

${ }^{1-4}$ Department of Periodontology, Saraswati Dental College Lucknow, Uttar Pradesh, India

Corresponding Author: Ruchi Srivastava, Reader, Department of Periodontology, Saraswati Dental College, Lucknow, Uttar Pradesh, India, Phone: +919793889594, e-mail: drruchi117@ gmail.com
}

junction (CEJ) with loss of periodontal connective tissue fibers. ${ }^{1}$ A survey showed that about $88 \%$ of people above 65 years of age and almost $50 \%$ of people between 18 and 64 years have recession in one or more sites. ${ }^{2}$ Currently, different mucogingival surgeries are available for root coverage. The basic techniques are pedicle grafts, free mucosal graft, subepithelial connective tissue graft, and membrane barrier-guided tissue regeneration technique.

Pedicle graft was the first periodontal plastic surgery procedure, proposed by Grupe and Warren, ${ }^{3}$ for root coverage. Pedicle grafts are based on the concept of moving donor tissue laterally, with its base attached to cover an adjacent defect. When the lateral movement is from both sides, mesial and distal to the defect, the flap is known as a double papilla flap. Double papilla flap procedure was first described by Wainberg as the double lateral repositioned flap and was refined by Cohen and Ross $^{4}$ as the double papilla flap. The width of the attached gingiva and the length and thickness of keratinized tissue adjacent to the recession site should be adequate. Indication for this technique is in isolated recession with adequate width of attached gingiva. Contraindications include lack of sufficient attached gingiva at the donor site or presence of any fenestration or dehiscence defect of supporting bone at the donor site. This article describes a case in which double papilla flap combined with amnion membrane was used for root coverage in Miller class II defect in mandibular canine. The amnion membrane is a preservable material derived from the human amnion and consists of a pluripotent cellular element embedded in a semipermeable membranous structure. It is used for various perioplastic procedures, because of its immunological preference and antimicrobial, mechanical, and pain-reducing properties. ${ }^{5}$

\section{CASE REPORT}

A 38-year-old male patient was referred to the Department of Periodontology at Saraswati Dental College, Lucknow, with the chief complaint of receding gums with hypersensitivity. The patient had a Miller class II recession defect on the mandibular right canine. The distance from the CEJ to the marginal gingiva in 43 was $3.5 \mathrm{~mm}$ (Fig. 1A). The patient had positive tactile and air blast sensitivity. Any etiology that may contribute to the progression of recession was identified and ruled out. 
Also, instructions for proper toothbrushing and oral hygiene were given to ensure maintenance before, during, and after therapy.

The procedure was explained to the patient, and informed consent was obtained. Proper evaluation of gingival biotype of donor areas was done by transgingival probing and found to be of the thick biotype. ${ }^{6} \mathrm{~A}$ root coverage with double papilla technique, utilizing amnion membrane was planned after Phase I therapy (after 4 weeks). After local anesthesia with $2 \%$ lignocaine hydrochloric acid (1:200,000 adrenaline), a V-shaped incision surrounding the recession defect was given (Fig. 1B). This allows the removal of a wedge of marginal tissue and provides a fresh wound surface for tissue approximation. A submarginal incision was then made on both adjacent teeth to leave a collar of marginal gingiva,
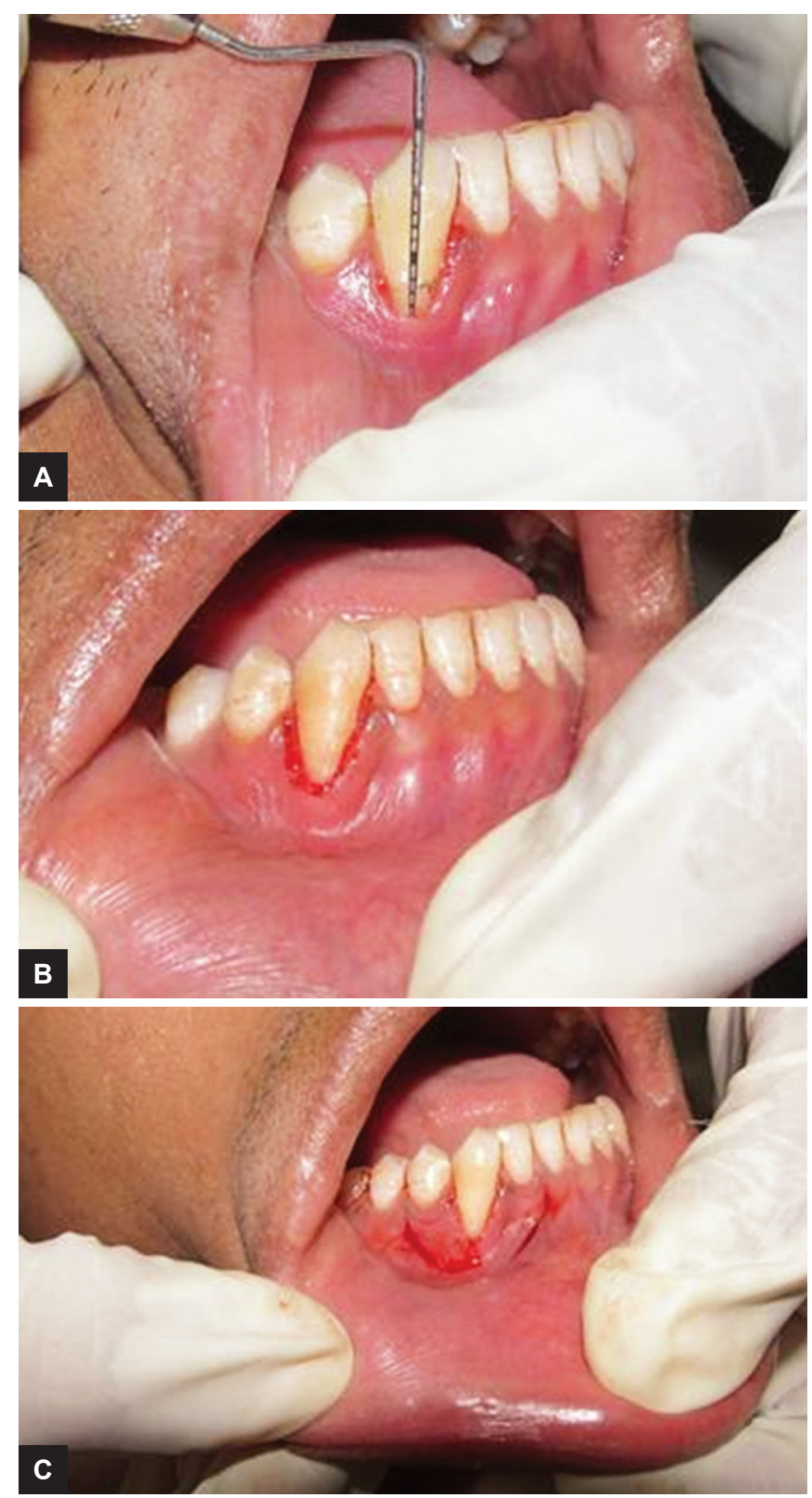

Figs 1A to C: (A) Preoperative recession in 43; (B) deepithelialization; and $(C)$ incision on both sides followed by mesial and distal vertical-releasing incisions (Fig. 1C). A split-thickness lateral sliding flap was raised from both adjacent teeth that extended apically beyond the mucogingival junction to release the flap sufficiently (Fig. 2A). After flap elevation, the exposed root surface was thoroughly planed with the help of a curette, and chemical root conditioning was performed using tetracycline for 5 minutes (Fig. 2B). The amnion membrane was placed and both pedicle flaps were sutured without tension followed by suturing of vertical incisions using a 4-0 silk suture (Figs 2C and D). Light-cured periodontal dressing (Barricaid, Dentsply) was applied at the surgical site (Fig. 3A).

Postsurgical instructions were given and the patient was placed on amoxicillin $500 \mathrm{mg} 3$ times a day for 5 days, aceclofenac $100 \mathrm{mg} 2$ times a day for 5 days, and chlorhexidine $0.2 \%$ twice a day for 4 weeks. The patient was asked not to chew or brush for the first 4 weeks. Ten days after surgery, periodontal dressing and sutures were removed and the surgical area was carefully irrigated with $0.2 \%$ chlorhexidine. Significant root coverage was obtained without any postoperative morbidity or recession to the adjacent teeth. Oral hygiene instructions were reinforced. A follow-up examination after 3 months revealed stable root surface coverage (Fig. 3B).

\section{DISCUSSION}

Gingival recession, especially within the esthetic zone, is of primary concern for patients and must be corrected if an esthetic smile is to be achieved. If untreated, gingival recession may progress to the point that it can compromise the prognosis of the tooth in question. Several innovations and modifications have been developed for surgical root coverage. The surgical technique that is undertaken depends on several factors like the size of recession defect, anatomy of defect site, localization in esthetic region, the presence or absence of keratinized tissue adjacent to the defect, the width and height of the interdental soft tissue and depth of the vestibule, or presence of frenula. In this case report, a double papilla graft technique with amnion membrane was used for successful root coverage. Although the double papilla graft technique is not a new procedure and has been used alone or in conjunction with connective tissue graft or other membranes, ${ }^{7}$ to the best of the authors' knowledge, no published literature is available of double papilla graft in combination with amnion membrane. Use of amnion membrane as guided tissue regeneration not only maintains structural and anatomical configuration, but also contributes to healing through reduction of postoperative scarring and subsequent loss of function, and 

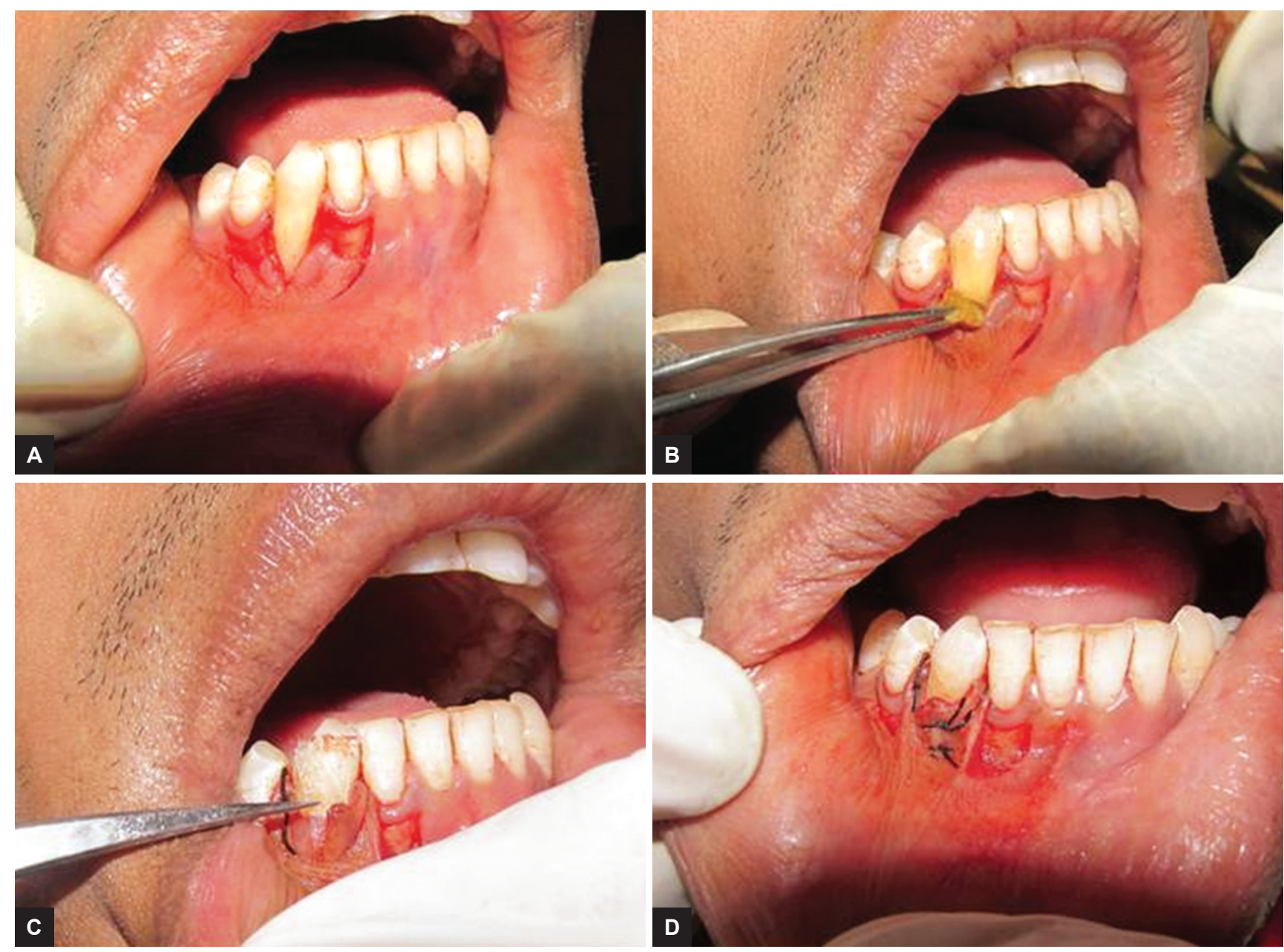

Figs 2A to D: (A) Double papilla flap; (B) root biomodification; (C) amnion membrane placed; and (D) sutures placed
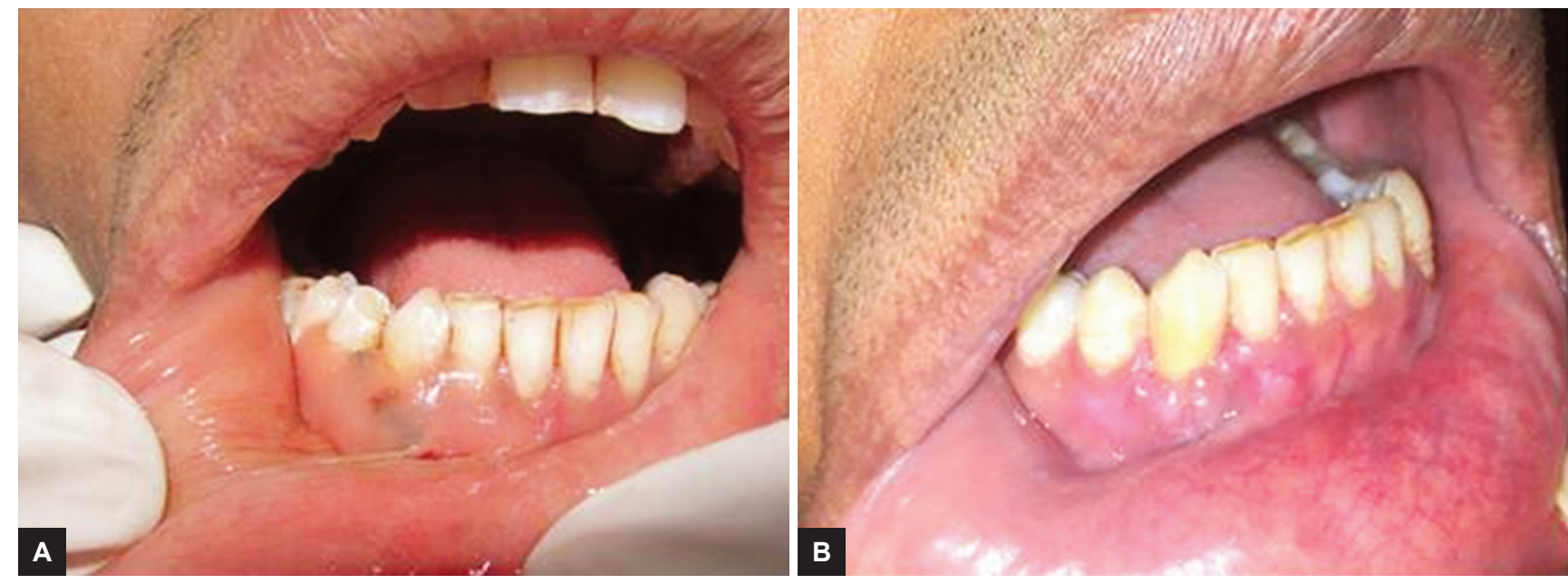

Figs 3A and B: (A) Barricaid periodontal dressing; and (B) three months postoperative in 43

also provides a rich source of stem cells. ${ }^{8}$ The extent and predictability of root coverage procedures are dependent on the quality of the vascularity that is maintained at the surgical site. The advantage of this procedure is that blood supply is maintained from the base of the pedicle and the surrounding areas of the recession defect. The gingival biotype of the adjacent flap is also an important aspect for root coverage outcome. ${ }^{9}$ The interdental papillary tissue should be thick enough at the area adjacent to the recession defect. A submarginal incision was given in the present case to preserve the integrity of the marginal gingiva and esthetic appearance at the donor site. The dimension of the flaps is critical for predictable root coverage, i.e., a wider pedicle will maintain increased blood supply to the flap. With the use of amnion membrane, the postoperative discomfort was minor and no 
second surgery or another surgical site was involved. ${ }^{10}$ The color of the pedicle graft matches well with the adjacent gingiva, and this procedure resulted in excellent esthetics with stable root coverage. As with any other technique, the disadvantages of this technique are possible bone loss, necrosis of the flap if it is too thin, and gingival recession at the donor site. Careful manipulation of the flap and adequate suturing is necessary to ensure proper healing in the desired position. Without adequate closure of both the papilla flap, separation can occur, resulting in exposure of the root surface.

\section{CONCLUSION}

The double papilla graft in conjunction with amnion membrane is an effective treatment modality for the management of isolated recession defect. It is a challenging and technique sensitive procedure to join the two delicate papillae and to avoid recession or bone loss at the donor site. However, this surgical technique resulted in highly satisfactory and complete root coverage of the recession defect.

\section{REFERENCES}

1. American Academy of Periodontology. Consensus report on mucogingival therapy. Proceedings of World Workshop in Periodontics. Ann Periodontol 1996 Nov;1(1):702-706.
2. Serino G, Wennstrom JL, Lindhe J, Eneroth L. The prevalence and distribution of gingival recession in subjects with a high standard of oral hygiene. J Clin Periodontol 1994 Jan;21(1): 57-63.

3. Grupe HE, Warren RF. Repair of gingival defects by a sliding flap operation. J Periodontol 1956 Apr;27(2):92-97.

4. Cohen DW, Ross SE. Double papilla repositioned flap in periodontal therapy. J Periodontol 1968 Mar;39(2):65-70.

5. Agarwal SK, Jhingran R, Bains VK, Srivastava R, Madan R, Rizvi I. Patient-centered evaluation of microsurgical management of gingival recession using coronally advanced flap with platelet-rich fibrin or amnion membrane: A comparative analysis. Eur J Dent 2016 Jan-Mar;10(1):121-133.

6. Kan JY, Morimoto T, Rungcharassaeng K, Roe P, Smith DH. Gingival biotype assessment in the esthetic zone: visual versus direct measurement. Int J Periodontics Restorative Dent 2010 Jun;30(3):237-243.

7. Shetty NJ. Double papilla repositioned flap for the treatment of isolated recession - A case report. Singapore Dent J 2013 Dec;34(1):25-27.

8. Shah R, Sowmya NK, Mehta DS. Amnion membrane for coverage of gingival recession: a novel application. Contemp Clin Dent 2014 Jul-Sep;5(3):293-295.

9. Shetty SS, Chatterjee A, Bose S. Bilateral multiple recession coverage with platelet-rich fibrin in comparison with amniotic membrane. J Indian Soc Periodontol 2014 Jan;18(1): 102-106.

10. Kolte R, Kolte A, Mahajan A. Assessment of gingival thickness with regards to age, gender and arch location. J Indian Soc Periodontol 2014 Jul;18(4):478-481. 